ob'sie noch eisenhaltig ist. Sollte dieses wider Vermuthen der Fall seyn, so leite ich noch eine halbe oder ganze Stunde lang Chlorgas in die Mischung, und trenne bierdurch die letzte Spur des vorhandenen Eisens jedenfalls, weinn man nur Snrge trägt, dafs noch Hohlensaures Zintoxyd in der Flüssigheit suspendirt ist. Die so gereinigte und filtrirte Lösung ist wasserhe!l und giebt durch vorsichtiges Fällen mit kohlensaurem Natron und der bekannten Behandlung des Niederschlags ein blendendweisses kohlensaures Zinkoxyd, und nach dem Glühen ein tadelloses weifses Zinltoxyd. Wiederholt machte auch ich die Erfahrung, dafs durch einen Ueberschufs des Fällungsmittels zugleich mit dem kohlensauren Zinkoxyd eine Verbindung desselben mit Íali niederfält, die, wie Welker bemtrkt, nach dem Glïhen, auch bei Abwesenheit des Eisens, bleibend gelb erscheint. Ob aber ein Zinkoxyd frei von Kohlensäure und zugleich so blendendweifs wie das kohlensaure Zinkoxy d darzustellen sey, möchte ich bezwcifeln; wenigstens kam mir weder in Apotheken, noch in chemischen Fabriken ein Zinkoxyd zu Gesichie, welches das nach der obigen Methode dargestellte an Weifse übertroffen habe *).

\title{
Weitere Erfahrungen über Zinkoxyd
}

\section{von}

W. Welcker zu Gedern.

In einer früheren Eingabe machte ich auf eine einfache Bereitungsart des reinen Zinlioxyds aufmerksam. - Weitere

-) Herr Marquart hatte die Güte, mir cine Probe seinea Präparato mitzutheilen, wclches ich für völlig tadellos crkannte. Die Keinheit der weifoen Farhe, velche man un dem kohlenwuren Zinkoxyd findet, wird enan wohl nie bci dem reinen on hohlensäure freien Zinkoxyd erłügen, $\quad N$ v. $E$. 
Frfahrungen, die ich in Betreff dieses Gegenstandes za machen Gelegenheit hatte, machen wir es nöthig, solche noch nachfolgen zu lassen, um den Hindernissen zu begegnen, die wohl hin und wieder bei Befolsung dieser Methode in den Weg treten, und wohl gar zum Mifstrauen eines glücklichen Gelingens fübren können. lch glaule daher keine unnütze Arbeit zu unternehmel, wenn ich das, worauf ich noch mit der Zeit aufmerksam wurde, und was mich, wia jch glaube, zu einem sicheren Ziele führte, hier noch nach. folgen lasse.

Bald nach der Darstellung des Zinhoryds nach meiner erwähnten Methode, nahm ich dasselbe Präparat wieder in Arbeit, nachdem ich hierzu das saure schwefelsaure Zinkoxyd aus Zündinaschinen gesammelt hattc. Ich neutralisirte die Lauge und behanılelte sie dann mit Gallustinctur. Dnvorhergesehene Arbeiten, und daher Hangel an Zeit, woran es mir stets regen häufiger Unterbrechungen laufender Geschäfte zu fehlen pflegt, waren einestheils die Ursache, warum ich meine Arbeit nicht liurz hinter einander vollendete, anderntheils glaubte ich auch durch diesen Aufschub nur zu gewinnen, indem ich dadurch eine höherc Oxydationsstufe des Eisens hervorzurufen hoffte, und so verstrichen denn mehrere Monate, bis ich durch den Defect dieses Praparats zur Herbeiholung meiner Zinllauge gemahnt wurde. Sie befand sich in einem grolsen steinernen 'Topf und hatte durch die Länge der Zeit eine derbe Schim. melhaut gezogen. Diese wurde abgenommen, die Lauge filtrirt; dan wie vorhin mit Eiweifs behandelt und erhitzt. Eines glücklichen Gelingens gewifs, gewahrte ich zu meinem Erstaunen, dafs der Verlauf dieser Arbeit nicht so glücken wollte, wie früher. Die Scheidung gieng awar von Statten, doch nur unvollständig. Das Eiweifs bildete hier mit dem Eisen ein so feines schwames Coagulum, dafs es sellst theilweise durchs Filter gieng, da hingegen bei meinem ersteren Versuch cin Collatorium angewandt werden honnte. Diese Verschiedenheit mufste mich nothwendiger- 
weise bald zu der Uebrrzeugung hringen, dafs hier eine Veränderung statt finda, und ich glaubte diese darin suchen zu müssen, dafs sich durch die Länge der Zeit der Gerbestoff in Gallussäure verwandelt hibe, und diese dem glücklichen Gelingen störend in den Weg trete. Es wurde da. her der Lauge nochmals Eir eils zugesetzt, erhitzt und dann filtrirt, allein sie lief dennoch zwar hlar, aber grünlich gefärbt durch, und man lonnte wegen des Opalisirens auf der Oberflüche derselben wahrnehnten, dafs sie noch etwas Eiweirs enthielt. - Es wurde uun zur Präcipitation mit kohlensaurem Natron geschritten. Der erste Niederschlag fiel mausfarbig aus; er wurde gesammelt und getrocknet, wobei seine Farbe in eine schmutzig weifse cibergieng, und dann geglüht.

Der zweite Niederschlag fiel dagegen volliommen weifs aus. Er wurde ebenfalls getıochnet und gegliiht. Fs zcigte auf diesen im mit Schwefelsüure aufgelösten Zustande GalPustinctur nicht dic mindeste Reaction. Blausaures Eisenoxydulkali fällte blausaures Eisenoxydul-Zinkoxyd vollkommen weifs, nahm indessen nach einigen Tagen uurerkennbar eine bläuliche Farbe an.

Nach diesen gemachten Beobachtungen schien es mir zwar immer weniger zweifelhaft, dafs ein glïckliches Gelingen der Arbeit nur der Reaction des Gerbestoff's zuzuschreiben seyn müsse; allein utn meiner Sache gewils zu seyn, suchte ich mir Gerbestoff und Gallussäure zu verschaffen, um mit beiden Versuche anstellen zu köanen.

Das Resultat werde ich hier folgen lasser.

\section{Erater Vereuch.}

\section{A. Mit Gallussäure.}

Nach der oben erwähnten zweiten Präcipitation crhaltenes, geglühtes Zinlioxyd wurde in verdümter Schwelelsiure aufyeläst, welches mit bedeutender Wäncentbindung verbunden war. Hierzu wurde eine tullösung von volliommeu oxydirtem schwefelsauren Eisen (chemiseh reincs schwe- 
feisaures Eisenoxydn! mit Salpetersäure behandelt, zum Trroknen gebracht u.s. w.) geģeben, and dann eine Gallussïureauflisung in Spiritus zugeselzt. - Die Reaction auf Eisoaoxyd erfolgte nur hüchst unmerhlich. Eiweifs zugesetzt, wurde dieses niedergeschlagen, und bildete durch Erhitzen ein mausfarbiges Gerinsel, welches durch das Filter geschieden und getrochnet eisce aschgrane Farbe annahm.

Da die Lauge nach Hinzutröpfeln von schwefelsaurer Eisenoxydaullösung noch Galiussïure anzeigte, so wurde nochmals Eiweif's zngesetzt und erhitzt.

(Auffallend, dars bier die Reaction auf Gallussäure voll. kommen energisch hervortrat, und bei der eisenoxydhaltigen Zinhauflösung die Gallussïure ihre Reaction versagte.)

Das erhaltene Gerinsel war jet/t nnch blasser von Farbe, die nach dem Trocknen eine schmutzig weilse war. D2 das Eiweis keine Veränderung mehr in der Farbe erlitt, so wurde nun die Lange, die zwar klar und durchsichtig erschien, aber eine grüne Farbe besafs, mittelst kohlensauren Natrons, und da dic Nenge derselben nicht bedeutend war, unvorsichtigerweise etwas in Ileberschu⿰s gefällt. Das erhaltene Oxyd fiel mansfarbig ans, und wurde durch Trocknen aschgrau. Geglitht et hielt es eine sebr gelbe Farbe, löste sich ohne Autbrausen, aber weit schwerer, als reines Zinhoxyd und mit gelber Farbe in verdünnter Schwefelsäure auf; - Gallustinctur reaginte sicht im entferntesten darauf, dagegen aber brachte blausaures Eisenoxydulkali augenblicklich einen völlig blauen Niederschlag hervor.

Eben so verhielt es sich mit der crhaltenen schwefelsauren Natronlauge, in der, obsteich sio mit Natron in Ueberschufs behandeit war, blausiaures Fisenoxydulkali au. genblicklich auf Eisenoxyd reagirte, dagegen aber Gallustinctar nhne alle Wirksamieit blieb. Die lauge besafs eine weingelbe Farhe. Ich werde anf diese Beobachtungen später wieder zurüctiommon, und gehe nun za feigendem Versuch über : 


\section{Zwoitor Versuch. \\ B. Mit Gerbest off.}

Nach der zweiten Präcipitation erhaltenes, gefoluhtes Zinkoxyd wurde in verdünnter Schwefeisäure aufgelöst, dieser Auflösung Eisenoxydauflösung zugesetzt und mit Gerbestoff behandelt. Die Reaction war höchst unbedeutend. Eiweifs zugesetzt und erbitzt, erhielt ich zwar ein, meinen ersteren Versuchen ähnliches Coagulum, welches durch ein Collatorium geschieden und ausgewaschen werden konnte, das aber nur eine schwärzlich blaue Farbe besafs. Die abfiltrirte Lauge reagirte noch anf Gerbestoff, und es wurde daher wiederum Eiweif's zugesetzt, wodurch sich ein Coagulum von ähnlicher Farbe bildete. Da nun die bekannten Reagentien keine Wirkung anf Fisen und Gerbestoff zeigten, die Iauge aber ein opalisirendes Ansehen wegen überschüssigen Eiweifses zeigte, so wurde nun zur Präcipitation mit kohlensaurem Natron geschritten, der erhaltene Niederschlag wohl ausgewaschen, getrocknet und geglüht. Nach dem Glühen erschien er gelb von Farbe, aber weniger hochgelb, als der vorhergehende, löste sich schwerer, wie reines Zinloxyd in verdünnter Schwefelsäure auf, äufserte auf Gallustinctur nicht die mindeste Feaction, eine ausgezeichnete indessen auf blausaures Eisenoxydultali.

Erstaunt iiber diese Erscheinungen, wurde alsbald ein zweiter Yersuch angestelit, und zwar in der Art, dafs ich zuerst etwas Eisenoxydauflösung in ein Medicinglas gols, dann Zinhoxyd zusetzte, und dieses in verdünnter Schwefelsänre aullöste.

Gcrbestoffauflösung zugesetzt zeigte zwar suf der Oberfläche einige Reaction, die jedoch beim Vermischen mit der ganzen Jaugenmenge wieder verschwand, und bei fortgesetztem Zungiefsen auch nicht vollständiger erfolgte. Es wurde Eiweifs zogesetzt und zwar mehr, als zur Verbindung mit Gerbestoff nöthig war. Die Lauge zeigte nach dem Erhitzen eine hellstrohgelhe Farbe, wurde filtrit, mit Gallustinctur reagirt, welche nicht die entfernteste Verän. 
derung gewahren liel's. Blausanres Eisenoxydulkali fällte blausaures Eisenoxydul.Zinkoxyd rollkommen weifs, und nahm erst nach mehreren T'agen eine bläuliche Farbe an. Hiermit noch nicht zufrieden, setate ich nun einer Zinloxydauflüsung, der Eisenoxyd zugesetzt worden war, Gallus. tinctur zu. Die Erscheinung war der obigen gleich; auf der Oberfläche zeigte sich anfangs ein schwärzlicher Schein, der beim Vermischen alsbald verschwand. Die hauge reichlich mit Eimeifs behandelt, warde dieses augenblicklich coa. gulirt, und sie exhielt nun eine strohgelbe Farbe. Die Reagentien zeigten mit dem vorhergehenden gleiche Erscheinungen, und das Oxyd wurde mit kohlensaurem Natron mit bläulicher Farbe gefällt. Da es hierdurch hinlänglich erwiesen ist, dafs das Eisenoxyd in einer sehr hartnäckigen. Verbindung in der Lauge noch enthalten war, so wurde die vollständige Fällung und ein weiteres Verfahren nicht fortgesetzt, uod ich gieng nun zu Versuchen mit Eisenoxydul über.

\section{Dritter Vesauch.}

\section{A. Mit Gallussäure.}

Obgleich es eine wohlbeliannte Sache ist, daf's die Eisenoxyd fällenden Galluspräparate auf das Oxydul lieine Reaction ausüben, so erschien mir dennoch auch ein Versuch hiermit aus besonderen Gründen nicht ganz überflüssig, um mich durch den Verlauf dieser Arbeit von allenfalls stattfindenden Veränderungen überzeugen zu können. Es wurde daher der mehrfach erwähnten Zinkoxydauflüsung schwefelsaure Eisenoxydulauflüsung zugesetzt. Gallussäure äufserte keine Veränderung darauf, selbst nachdem hiermit die Lauge eine Nacht hindurch stehen geblieben war. Als des andern Tags Eiweifs zugesetzt, wurde dieses sogleich gefällt. An dem Rand der Flüssigkeit zeigte sich ein unterbrochener blauer Ring. Beim Vermischen erhielt das Ganze einen hellblauen Schein. Es wurde nun erhitzt, wobei allmählig eine mehr blaue Farbe hervortrat, die bei längerem Er. 
bitzen wieder verschwand. Die Lange filtrirt erschien vollkommen klar und ohne Farbe. Durch blausaures Eisenoxydulkali niedergescblagen, erscbien der Niederschlag vollkommen weils, wurde aber nach mebreren Thgen blau. Sallustinctur blieb ohne Wirkung, dagegen aber zeigte Eisenoxydauflösuug augenblicklich Gallussäure an. Bemerkenswerth ist, dafs diese Reaction des Eisenoxyds vor dem Erhitzen nicht herrortrat.

Da nur durch das allmählige Blauwerden des Niederschlags mittelst blausauren Kali das Eisencyydul in einer chemischen Verbindung in der Lauge enthaltend erwiesen war und sich teine weitere Veränderungen zeigten, so wurde dieser Versuch als beendigt angesehen.

\section{B. Mit Gerbest off.}

Gerbestoff zeigte von dem Vorhergehenden im Wosentlichen kein verschiedenes Verhalten.

\section{Vierter Versuch.}

Mi: Eisenoxyd- und Oxydullösung.

A. Mit Gallussäure.

Schwefelsaurer Zinkoxydanflösung wurde ein Gemisch ron schwefelsaurem Fisenoxydul mit Oxyd zugesetzt und die Lauge nit Gallussäure behandelt. Die Färbung trat hier bedeutender hervor, als mit beiden Fisenpräparaten allein. Eiweirs wurde sogleich niedergeschlagen, und nahm eine dunkelblaue Färbung an. Durch das Aufwallen gieng dieselbe in eine schmutzig weifse über. Die Laage erschien nach c.em Filtriren wasserhell. Blausaures Ifali lieferte einen vellkommen weifsen, mit der Zeit blau werdenden Niederschlag. Eisenoxydauflösung reagirte stark anf Gullussäure. Dite wasscrklare Lauge nochmals nit Eiweifs behande?t, schlug letzteres wieder gethibt nieder. Es wurde jetzt ein Versuch ohre Wärmeanweniung mit Eiweils gemacht, da jedesmal hierdurch eine Farbenveränderung eintrat, allein 
wegen des rorhandenen Fiweifses konnte die tauge n:cht filtrirt werden, und es mufste daher zuvor durch Erhitzen wiederum coagulirt werden. Die Lauge war nun sebr eingeengt, Eiweil's wurde denohnģeachtet jetzt nicht mehr gefällt, äufserte jedoch die Wiriung; dafs lieselbe nun eine violettblaue Farbe annahra. Eisenoxyd zeigte darin noch Gallussïure an.

\section{B. Mit Gerbestoff und den vorhergehenden Reagentien.}

Gerbestoff änfserte anf die Lauge in Berücksichtigung des darin enthaltenen Fisenoxyds ebenfalls rur eine höchst unvollständige Reaction. Dieselbe mit Eiweifs geschïttelt, verdichte sich das Ganze und nahm eine blaugrüne Farbe an. Coagulirt und filtrirt, hatle die Lauge ein grünliches Anseben. Es wurde dieser nochmals Eiweifs zugesetzt und erhitzt, letzteres coagulirte und die Lauge erschien nun vollkommen farblos, reagirte indessen auf Gerbestoff. Blausaures Eisenoxydulkali lieferte einen den vorhergebenden vollkommen ähnlichen Niederschlag, der erst mit der Zeit blan erschien.

\section{Mit Gallustinctur u. s.w.}

Gallustinctur zeigte ror den beiden vorhergehenden Versuchon keine Verschiedenheit.

Bei den bis hierher stattgefundenen Versuchen verdient tesonders berücksichtigt zu werden, wie auch aus dem Gesagten hervorgeht, dafs das hierzu angewendete Zinkoxyd zwar dem Aeufseren nach ein schönes Präparat, aber dennoch nicht frei ron Eisen war, und $z$ war in einer so constanten Verbindung, dafs selbst die empfindlichsten Heagentien ihre Wirksamkeit versagten. Ueber die Ursachen dieser abweichenden Erschcinungen werde ich mich später aussprechen, nacbiem ich die nachfolgenden Versuche werde mitgetheilt haben, $\mathrm{nm}$ auch aus dem Verhalten dieser meine 
Bemerkungen über die vorhergehen:len deutlicher beweisen za können.

\section{Fünfter Versuch.}

Es wurde Zinh in verdtinnter Schwefelsäure mittelst Wärme bis zur Nenłralisation aufgelöst, die Lauge filtrirt und damit folgendermafsen rerfahren:

\section{A. Mit Gallussäure.}

Die Lauge, mit Gallussäure versetzt, enlitt, nachdem sie eine Nacht hindurch gestanden hatts, heine weitere Veränderung, als difs sic einen nur etwas grüntichen 8chein anna:bm. Mit Eiweifs bebandelt, wurde dieses mit bellgrauer Farle gefällt, und das überschüssige durch Erhitzen coagulirt. Die abfiltrirte Lange reagirte mit Eisenoxydanflosung noch auf Gällussäure, sie wurde daher nochmals mit Eirreifs bohandelt, welches aber jetzt unbedeutend gefält wurde. Das überschüssige Eiweifs wurde durch Erhitzen coagulirt, worauf die Lauge nach dem Filtriren mit Eisenoxydauflösung nicht mehr auf Gallussäure reagirte. Es wurde in einem Probegläschen etwas Lauge mit blausaurem Eisenoxydullkali gefällt (blausaures Eisenoxydkali besafs ich mieht) und solches der Finwirknng der Luft hingestellt. Selbst nach neun Tagen zeigte sich hierin nicht die mindeste Farbenweränderung. Ein kleiner Theil der Lauge wurde mit Chlor behandelt, welches Eisen darin gewahren liefs. Die noch übrige I,auge wurde nun mittelst hohlensauren Natrons gefüllt. Das erhaltene Oxyd fiel sehr schön weifs aus, und belielt auch dieses schöne Ansehen selhst nach sehr starken Glühen. Eiwas von diesem Oxyd in verdünnter Schwefelsäure aufgelöst und mit blausaurem Eisenexydulkali niedergeschlagen, behielt mehrere Tage seine weilse Farbe, und erst nach acht Tagen liefsen sich blaue Theilché; in dem Oxyd uni an den Seitenflächen des Glases in dem daran hängenden Niederschlage bemertien.

Die erbaltene basische schwefelsaure Natronlauge virde mit blausaureın Eisenoxydulkali reagirt, welches nich die 
mindeste Veräıderung hervorbrachte. Mit einigen Tropfen Salpetersäure zersetzt, um allenfalls darin vorhandenes Eisenoxydul in Oxyd zu verwandeln, und in einem CpodelJolsgläschen erwärmt, erhielt ich nach einiger Zeit ein sehr schönes Sälzchen, zuin Theil regelmäfsige Kreuzchen bildend, die sich nach aufsen federortig ausbreiteten. Ueber seine Bestandtheile kann ich nichts sagen, da die erhaltene Menge zu unbedeutend war, um Versuche damit haben ansteller zu hönnen.

Mit Hydrothionammoniak zeigte die schwefelsaure Natronlauge heinen Niederschlag. Mit Gallustinctur reagirt, zeigte sich in der concentrirten Lauge eine sehr milchige, etwas dickliche Trübung, die bei fortgesetztem Zugiefsen von Gallustinctur wieder verschwand, dann wieder in der Art hervortrat, dafs sich auf dem Boden des Glases ein lockerer salzartiger. Bodensatz, und auf der Oberfläche der Lauge deutlich lileine Irryställchen zeigten, die bei Zugiessen von Wasser wieder aufgelüst wurden.

Ein Kleiner Theil der Lauge mit Schwefelsäure neutralisirt, in zwei Probegläschen gethan, wovon das eine etwas basisch, das andere etwas sauer reagirte, bewirhte Gallustinctur diesen schmutzig weifsen Bodensatz, der nach $\mathbf{A b}$ giefsen der überstehenden Lauge in kaustischem Ammoniak sich leicht, mit rother Farbe, auflüste, und durch Salzsïure wieder gefältt wurde. Die Laugenmenge war zu unbedeutend, um sie mit Gallusinctur zu behandeln und den erhaltenen Niederschlag weiter untersuchen zu köınen. Doch wurde ich hierdurch aufner'isam ouf meine erstere Glaubersalzlauge, wovon sich noch die Mutterlauge vorfand. Diese lieferte mit Gallustinctur einen schnutzig weifsen Niederschlag, und die überstebende Lavge nahm eine dunkeigrüne Farbe an. Dieser Nutterlauge etwas Eisenoxydanflösung zugesetzt und mit Gallustinctur reagirt, entstand augenblichlich ein, wahrscheinlich wegen ungebundenen Natrons, castanienbrauner Niederschlag. 
Die ganze Menge dieser Lauge wurde sun nittelst Gal. lustinctur gefällt, wozu nur wenig nöthig war. Der crbaltene Niederschlag nahm eine bräunliche Farbe an, war ius feuchten Zustande von sehr voluminöser Beschaffe!? eit, beirn Erwärmen nahm derselbe eine grüne Farbe an, die bei fortgesetztem Eintrocinen immer dunkler wurce. Getrocknet blieb nur wenig zum Versuche übrig. In einem Tiegelchen geglïht, bildete die oberste Fläche einen dunkelgrauen Ueber. zug, unter welchen das Uebrige eine aschgraue Futs: besafs, und hepatisch rorh. An den Seiten des Tiegels befanden sich gelbgrünliche Streiten. Einige Gran dieser Masse in Salzsäure aufgelöst, entwickelten einige Gasblïschen mit hepatischem Geruch. Mit Ammoniak entstand cin liseiner Niederschlag, der auf dem Filter zu einem gelben Körnchen eintrocknete, sich nicht in kanstischem frali auflöste, und mit Gallussäure betropt einen schwarzen Fleck bildete. Das Uebrige des Tiegelinhalts wirde in Salzsüure aufrelöst. Mit Ammoniak in Ucberschufs behandelt, entstand ein unbedeutender Niederselslag, der zu gering war, um ihn rom Filter. zu nehmen. Er wurde darauf mit taustischer Lauge ausgelaugt, worin mit Salzsäure keine Trübung erfolgte. Das Filter mit Gallussäure betropft, wurde an der Luft schwarz. Die abfiltrirte ammonialalische Lauge mit Salzsäure neutralisirt, liefs nicht die mindeste Trübung gewahren.

Hiernach bestand der mit Gailustinctur erhaltene Niederschlag, der nur wenig ausgewaschen worden war, aus Schwefelsäure, Natron, Eisenoxydul und gefälltem Gerbestoff, welcher letzlere theilweise eine Nleduction der Schwe. felsäure zur Folge hatte.

Die von diesem Niederschiage abfiltrirte Lauge liesals cine dunkelgrune Farbe, und weiter mit Gallhastinctur vey. setzi, sehluger: sich angenblicklich gelbgrüliche Krystältchen nieder. Diese nt Alkohol ausgewaschen behielten eine schmutzig weifse Farbe. Salzsaurer Bary bewirite in der Aufliosung derselben sinen weilsen, in zuyesetzter Saipeter-

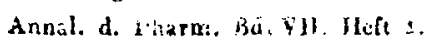


sn̈ure unauflöslichen Niederschlag. Eisenoxydaufl̈sung reagirte aut Gallussäure, was ich jedoch nach wiederholtem Auswaschen mit Alkohol nicht wieder bemerken konnte Blapsaures Eisenoxydulkali zeigte heine Reaction. Das Salz wurde nun in einem Tiegel geglüht. Fs blieb eine weifse Salzmasse, dic in Wasser aufgelöst, mit Salzsäure neutralisirt, einige Gasbläschen entwickelte. Mit Ammoniak zeigte sicb ein geringer im Ueberschufs von Ammoniak unautlöslicher Niederschlag. Nach dem Sedimentiren desselben wurde die überstehende Flüssigkeit abgegossen, dem Niedersehlage einige Tropfen Salpetersäure zugegeben und erhitzt. Mit Gallussäure reagirt, liefs kein Eisen gewahren. Fs wurde nun noch ein Versuch mit einer andern Probe mit kaustischem Kali gemacht, allein auch dieses löste den Niederschlag nicht auf, und es konnte daher dieser für nichts andars a t. kannt werden, als Magnesia.

Die obfiltrirte ammonialalische Lauge liefs mit Essigsäure heine Trübung finden. Mit salzsaurem Baryt lieferte die mit Salzsëure neutralisirte Lauge einen in Salpetersäure unaullöslichen Niederschlag.

Die untersuchte Salzmasse bestand demnach aus Schwefelzäurc, Natron und einer Spur Magnesia, welche letztere ron der Schmelztiegelmasse herrühren mochte.

Mein Bestreben war, mich $2 u$ überzeugen, ob nicht Eisen oder Zinloxyd in den beiden letzten Versuchen vorhanden war.

Die übrige Mutterlauge auf dieselbe Weise bebandelt, liefs mittelst Hydrothionammoniak bedeutend Eisen finden. Mittelst bohlensaurem Kali tabe ich gefunden, dafs es in oxydirten und oxydulirten Zustande darin enthalton war.

\section{B. Mit Gerbestoff.}

Die Lauge wurde gleichreitig mit der mit Gallussäure, mit Gerbestoff ve:setzt. Er zeigte beinahe keine Reaction, und nachdem sie so gegen 4-5 Tage gestanden hatte und 
nichl die mindeste Veränderung gewahren liefs so wurden Versuche hiermit nicht weiter fortges"tzt.

$$
\text { Sechoter Verauch. }
$$

Zink wurde in revdünnter Schwefelsäurc gegen das Ende durch Würme unterstützt, bis zur Neutralisation aufgelöst, die erhaltene Zinklange filtrint, reagirte mit den behannten Meagentien (worunter jedoch nicht blausaures Eisen. oxydkali war) nicht auf Eisen. Wer Lauge Eisenoxydauflo. sung zugesetzt, trat hier diese Reaction augenblicklich ein. Dafs indessen Eisen darin in oxydulirtem Zustande enthalten, ist schon oben erwähnt worden. Das Oxyd mittelst kohlen. sauren Natrons gefällt, fiel sebr scbön weifs aus, und behielt auch diese weilse Farbe noch nach heftigem Glühen. Im avfgelösten Zustande brachte dasselbe mit Galluspräparaten keine Reaction hervor; blausaures Eisenoxylulkali zeigte auch nach 10 Tagen nicht die mindeste Farbenveränderung.

Nachdem ich nun bis hieher mit meinen Versuchen gokommen war, und die Ergebnisse derselten beleuchtete, konnte mir nicht entgehen, meine besondere Aufmerksamkeit auf die im ersten Versuche $A$. beschriebene, Eisenoxyd entbaltende schwefelsaure Natronlauge zu richten, die bei dem Ueberschufs von Natron wohl nichts anders, als eine Trippelverbindung aus Schwefelsäure, Natron und Eisenoxyd seyn Konnte,

Ist nun diese Verbindung als erwiesen anzunehmen, so mufste mir nichts wahrscheinlicber seyn, als dafs eine solche Trippelverbindung auch in der scliwefelsauren Zinklauge statt finden und die Ursache seyn müsse, warum die ange* wandten Reagentien ihren Dienst versagten. Folgende Ver. suche scheinen denn auch dieses aufser allen Zweifel zu setzen:

a) Das im ersten Versuch $A$. erhaltene Zinkoxyd wurde in verdünter Salzsäure aufgelöst, mit salzsaurer Bzrgtauflösung reagirt, wodurch keine Trübung erfolgte.

b) Nach dem zweiten Versuch B. erhaltenes Zinkcxyd 
auf dieselbe Weise behandelt, zeigte sehr bedeutend Schwefelsäure an.

c) Ehen so verhielt es sich auch bei dem im fünften Versuch 1 . erhaltenen Zinlioxyd.

d) Defsgleichen mit dem im sechsten Versuch erhaltenen.

Bei dem im ersten Versuch $A$. erhaltenen Zinkoxyd war demnach diese Trippelverbindang als schwefelsaure Eisennatrontrippel rerbindung in die Glanbersalzlauge übergetre. ten. Hieraus möchte wohl mit vieler Wahrscheinlichkeit zu entnehmen seyn, dals die nach diesen Versuchen erwiesene Trippelverbindung, bestehend aus Schwefelsäure, Zinkoxyd und Eisenoxyd, mit dem letzteren eine weniger constnnte Verbindung einzugehen vermöge, als mit den niedrigeren Oxydationsstufen des Eisens.

Diese Beobachtang war auch hauptsächlich die Ursache, und möge hier zur Entschuldigung dienen, dafs ich mich hin und wieder mit Versuchen beschäftigte, die, ohne den Grund zu erratben, zu welchem $Z$ weck sio angestellt viur. den, für üherflüssig erscheinen mochten.

Dals man auf diese Trippelverbindung bisher wenig aufmerksam war, geht aus den $z$ weifelhaften und getheilten Meinungen über die häufig statt findende gelbe Farbe des Zintoxyds hervor. Ebenso geht auch aus diesen Versuchen hervor, dnfs diese nur der Anwesenheit ron Fisenoxyd zuzeschreiben sey, Zinhoxyd in reinsten Zustand sich be: dem heftigsten Glïhen stets eine weilse Farbe eshalte; and dafs diese auch selbst bei einer Trippelverbindung in einer niedrigen Oxydationsstufe des Eisens selbst nach heftigem Glühen noch der Fall sey. Auch scheint mir bemerkenswerth, defs diese niedrige Oxydationsstufe in einer Trippelverbin. dung, worin sie gewöhnlich erhalten wird, selbst nach längerem Ausgesetztseyn der atmosphärischen Luft und der Wärme nicht in eise höhere übergebe, und dafs dieses selbst bei einesn solchen Oxyd während des Glühens nicht der Fall ist. Hierin liegt auch meiner Ansicht nach die Ursache, 
warmm man haufig ein so verschiedenartiges Verhalten des Zinkoxyds nach dem Glühen gewahrt, und die Ursache seiner Farbe einer mehr oder minder angewendeten Hitze zuschreibt. Der Grund hiervon licgt wohl gröfstentheils in der Reinheit der hierzu angewendeten Schwefelsäure; die wohl oft nicht die nämliche Qualität seyn mag, und durch ihre Verunreinigung mit Salz- und Salpetersäure, je nach der Menge derselben, eine mebr oder mindere Menge Eisen in Oxydoxydul verwandelt. Hier würde das Oxydul seine Stelle als Trippelverbindung einnehmen, und das Eisenoxyd in ungebundenem Zustande das Zinhoxydpräparat gefärbt darstellen. So kann denn gleichzeitig ein Priiparat Eisen als Trippelverbindung in oxydulirtem und in ungehundenem oxydirten Zustande enthaiten. Ich bin selir geneigt zu glauben, dafs das bisher verbrauchte Zinkoxyd in den meisten Fillen eine solche Trippelverbindung war, selbst wein es auch ein sehr schönes äufseres Ansehen besessen habe, und dafs diese Verbindung, bei ihrer Mächtigkeit, den hierzu angewandten Reagentien zu widerstehen, eine Täuschung in der Reinheit des Präparats häulig herbeigetührt habe. Diese Verbindung in den viedrigen Oxydationsstufen ist so mächtig, dafs selbst Salpetersäure nicht ausreichen möchte, utn eine höhere Oxydationsstufe herbeizuführen. Ein mächtigeres Mittel findet sich jedoch an dem Chlor, das vregen seines so energischen Bestrebens in Berihrung mit leicht oxydirbaren Metallen oder deren niedrigen Oxydationsstufen das Wasser schneli zu zerlegen, und durch den dadurch frei werdenden Sauerstoff die Oxydation herbeizuführen vermag. In. diesem mächtigen Bestreben findet sich denn auch das Mittel, diese constante Verbindung aufzuheben und nun eine böhere Oxydationsstufe des Eiseas herbeizuführen. Meine Versuche hierüber folgen.

Nachden ich cine schwefelsaure Zinkoxydlauge ctwus basisch gemacht hatte, wurde Chlorgas durchgeleitet. Es fälle dadurch etw as Eisenoxyd, was sich bei längerem Durch- 
strömen wieder verminderte. Es findet hierbei Oxydation und Reduction statt, je nach dem Durchstrëmen des Chlor. gases. Ich liefs viel Chlorgas durchströmen. Die Lauge worde, nachdem sie filtrirt, zur Trockne gebracht, wobei sich kein Eisenoxyd mehr ausschied. Aufgelïst wurden Ver. suche damit angestellt :

\section{A. Mit Gallussäure.}

Die Reaction erfolgte jetzt sogleich. Mit Eiweifs hebandelt und erhitzt warde das Eisen in das Coagulum roll. hommen eingewickelt, und das erhaltene Oxyd wurde jetzt weder als Trippelverbindung noch eisenhaltig befunden. Bäthlich ist es indessen, eine solche mit Chlorgas behandelte Lauge noch cinige Zeit der atmosphärischen Luft auszusetzen, um das Gelingen zu vergewissern.

\section{B. Mit Gerbest off.}

Hiermit wurde ebenwohl der Zweck vollständig erreicht, und ist wie bei dem Vorhergehenden auch hiermit zu ver. fahren.

Hiernach wäre also dieses Verfahren, obgleich es zum Ziele führt, dennoch wegen des hierbei anzuwendenden Chlorgases, mit einigen Beschwerlichkeiten verknüplt. Man Lann indessen diesen durch die Anwendung von Hydroshlorsäure mit dem besten Erfolg ausweichen, wie ich mich hiervon durch Versuche hinlänglich überzeugt habe.

Zink wurde in Hydrochlorsäure, gegen das Ende durch Wärme unterstützt, aufgel8st. Der filtrirten Lauge destillirtes Wasser zugesetzt, bildet sich ein Niederschlag, den jch für Zinkhyperoxyd (?) halte, und der wesentlich dazu beiträgt die Arbeit zu beschleunigen, indem dieses Hyperoxyd durch das Bestreben der hierzu angewandten Reagentien, sich mit dem Eisen zu varbinden, eine Reduction erleidet, und hierdurch die schnelle Oxydation des Eisens herbeiführt.

Es wurden Versuche angestellt: 


\section{A Mit Gallussäure.}

Es erfolgte mit Gallussäure anfänglicb heine Reaction, die sich aber nach mehreren Stunden durch hellblaue Färbung der Lauge einstellte, immer mehr und mehr in das Dunklere überging, so dafs binnen eines Tages die Reaction vollstündig erfolgt war und die Lauge nun mit Eiweirs behandelt werden konnte. Das Oxyd fiel ausgezeichnet weifs aus und zeigte gegen Heagention weder Salasäure noch Eisen an.

\section{B. Mit Gerbestoff.}

Mit Gerbestoff erfolgte anfünglich ebentalls heine Reaction, die sich aber schon nach einer Viertelstunde voll. ständig einstellte, so dafs schon jetzt die Rehandlung mit Eiweifs vorgenommen werden konnte. Das erhaltene Oxyd zeichnete sich durch eine weifse Farbe aus und verhielt sich gegen Reagentien dem vorbergehenden gleich.

Hiernach ist also auch die Anwendung von Chlor beseitigt, und dieses Verfahren wegen seiner Einfachheit dem. mit Schwefelsäure weit vorzuzieben.

Die Anwendung von Gallussäure und Gerbestoff' führen beide zum Zweck, doch zeigt sich deutlich, dafs die Keaction der Gallussäure der des Gerbestoffs etwas nacbsteht, wie ich dieses bei meinen Versuchen wahrzunehmen Gelegenheit hatte.

Mein 'Zweifel in Betreff der Anwendung beider Präparate ist hierdurrh gehoben, und die Anwendung der Gallustinctur, die beide in gebundenen Zustande enthält, tritt daher wegen ihrer leichtern Darstellung an deren Stelle 